\title{
Responsive Cortical Stimulation for the Treatment of Epilepsy
}

\author{
Felice T. Sun,* Martha J. Morrell,* and Robert E. Wharen, Jr. ${ }^{\dagger}$ \\ ${ }^{*}$ NeuroPace, Mountain View, California 94043, ${ }^{\dagger}$ Mayo Clinic College of Medicine, Department of Neurosurgery, Jacksonville, \\ Florida 32224
}

\begin{abstract}
Summary: Epilepsy is a common chronic neurological disorder affecting $\sim 1-2 \%$ of the population. Despite the available treatment options (pharmacotherapy, surgery, and vagus nerve stimulation), a large percentage of patients continue to have seizures. With the success of deep brain stimulation for treatment of movement disorders, brain stimulation has received renewed attention as a potential treatment option for epilepsy. Responsive stimulation aims to suppress epileptiform activity by delivering stimulation directly in response to electrographic activity. Animal and human data support the concept that responsive stimulation can abort epileptiform activity, and this modality may be a safe and effective treatment option for epilepsy. Responsive stimulation has the advantage of specificity. In contrast to the typically systemic administration of
\end{abstract}

pharmacotherapy, with the concomitant possibility of side effects, electrical stimulation can be targeted to the specific brain regions involved in the seizure. In addition, responsive stimulation provides temporal specificity. Treatment is provided as needed, potentially reducing the likelihood of functional disruption or habituation due to continuous treatment. Here we review current animal and human research in responsive brain stimulation for epilepsy and then discuss the NeuroPace RNS System, an investigational implantable responsive neurostimulator system that is being evaluated in a multicenter, randomized, double-blinded trial to assess the safety and efficacy of responsive stimulation for the treatment of medically refractory epilepsy. Key Words: Epilepsy, responsive stimulation, neurostimulation, device, closed-loop.

\section{INTRODUCTION}

Epilepsy is a common chronic neurological disorder affecting $\sim 1-2 \%$ of the population in the United States. ${ }^{1}$ Despite the available treatment options (pharmacotherapy, surgery, and vagus nerve stimulation), a large percentage of patients continue to have seizures. Pharmacotherapy is the standard of care for epilepsy patients. Nevertheless, $\sim 30 \%$ of patients continue to have seizures or experience medication-related side effects such as nausea, dizziness, drowsiness, and weight gain., ${ }^{2,3}$

Surgery is an option for some patients. Although anterior temporal lobectomy in persons with mesial temporal sclerosis can achieve a 1- to 2 -year remission in $\leq 85 \%$ of cases, ${ }^{4}$ resection of a well-defined neocortical lesion achieves 1 -year seizure remission in only $56 \%$. In addition to the typical risks associated with a craniotomy and general anesthesia, complications of surgery may include memory, language, visual, sensory, or motor deficits. ${ }^{4}$ Moreover, many persons are not candidates for surgery because the epileptogenic region involves func-

Address correspondence and reprint requests to: Martha J. Morrell, M.D., Chief Medical Officer, NeuroPace, Inc., 1375 Shorebird Way, Mountain View, CA 94043. E-mail: mmorrell@ neuropace.com. tional cortex and the risks of postoperative neurological deficits are too high.

Vagus nerve stimulation (VNS) is another option for patients with medically refractory epilepsy. In the controlled trials leading to market approval, adults with partial onset seizures treated with optimal VNS stimulation parameters had a median seizure reduction of $25-30 \%$, and $<2 \%$ experienced seizure freedom at 1 year. ${ }^{5}$ Given the limitations of currently available therapy options, new approaches for treating epilepsy are necessary.

Recently, with the success of deep brain stimulation (DBS) for treatment of movement disorders, brain stimulation has received renewed attention as a potential treatment option for epilepsy. Ultimately, electrical stimulation may be more tolerable than conventional therapy options such as pharmacotherapy and surgery because stimulation parameters can be adjusted for each individual patient and stimulation can be turned off if adverse events occur. The earliest report of applying electrical stimulation to the brain to treat seizures in humans is by Penfield and Jasper in $1954 .{ }^{6}$ In their acute experiments, they observed that in some cases focal electrical stimulation of the exposed cortex resulted in a flattening of the local electrocorticography (both normal rhythms and 
spontaneous epileptiform discharges). Building on these initial experiments, numerous studies have been published over the last three decades that examine the safety and efficacy of open-loop and closed-loop, focal and non-focal electrical stimulation for the treatment of epilepsy. $^{7-9}$

In the first chronic brain stimulation trials for epilepsy, electrical stimulation was delivered in an open-loop manner; that is, therapy was delivered according to a predefined schedule, independent of physiological activity. These studies targeted deep brain structures remote to the epileptic focus, such as the cerebellum, thalamus, and basal ganglia. ${ }^{10-12}$ More recent open-loop stimulation paradigms have also included stimulation into the epileptogenic region, such as the mesial temporal structures and primary motor cortex. ${ }^{13-15}$ A review of openloop DBS for epilepsy is provided in this issue by Halpern et al. ${ }^{16}$

In contrast to open-loop stimulation, closed-loop or responsive stimulation aims to suppress epileptiform activity by delivering stimulation directly in response to the electrographic activity. Due to the technical complexity of developing an integrated system that provides realtime electrographic analysis and automatic delivery of responsive stimulation, few centers have specifically tested the efficacy of responsive stimulation. Here we review current animal and human research in responsive brain stimulation for epilepsy, and then discuss the RNS system by NeuroPace (Mountain View, CA), an implantable responsive neurostimulator system that is currently being evaluated in a multicenter, randomized, doubleblinded trial to assess the safety and efficacy of responsive stimulation for the treatment of medically refractory epilepsy.

\section{RESPONSIVE STIMULATION THERAPY FOR EPILEPSY}

Experiments in animal models and in vitro neurophysiology suggest that modulating the specific timing of stimulation might be more efficacious than continuous, scheduled, or random stimulation. In the 1980s, Psatta ${ }^{17}$ delivered low-frequency $(5 \mathrm{hz})$ stimulation to the caudate nucleus in response to spontaneous spikes, which were detected at the chronic epileptogenic focus (induced by application of cobalt on the exposed neocortex). He reported that spike depression occurred immediately after the onset of stimulation, and that responsive stimulation was more effective than random stimulation to the caudate nucleus. In the 1990s, Durand and colleagues ${ }^{18-20}$ demonstrated success in suppressing spontaneous interictal bursts in vitro by providing responsive stimulation directly in the epileptogenic region. Their result suggested that the mechanism for suppression is an inhibitory polarization caused by the transmembrane currents generated by the applied pulse. These trials in animals laid the groundwork for responsive stimulation therapy for epilepsy.

Early experiments to demonstrate the efficacy of responsive stimulation in humans were acute trials of stimulation performed on patients already undergoing intracranial mapping to localize seizure onsets prior to epilepsy surgery. During these procedures, electrical stimulations are applied to the cortex to identify functional and epileptogenic regions; the stimulus intensity is progressively increased until there is a clinical alteration or until an after-discharge is produced. Although afterdischarges are not the same as spontaneous epileptiform activity, they are similar in morphology to spontaneous discharges and can evolve into clinical seizures.

Lesser et al. ${ }^{21}$ demonstrated that brief cortical stimulation was effective in terminating after-discharges in an acute setting. Stimulation amplitude, frequency, and pulse-width parameters were identical to the mapping stimulation used to initiate the after-discharge. The terminating stimulus was of a short duration $(0.3-2 \mathrm{~s})$, however, whereas the mapping stimulation was typically $4-5 \mathrm{~s}$ in duration. Notably, they determined that longer burst durations were less likely to stop after-discharges than shorter durations; for a 1-s increase in stimulation duration, there was a decreased efficacy of $40 \%$. In a subsequent experiment, Motamedi et al. ${ }^{22}$ identified that stimulation was most effective in terminating after-discharges if they were applied early and at the primary electrodes (where the mapping stimulus was delivered).

To provide stimulation in response to spontaneous epileptiform activity, an integrated system that performs real-time electrographic analyses, and automatically delivers stimulation in response to detected events is required. In the first trials of responsive stimulation of spontaneous epileptiform activity, large nonimplantable bedside prototype systems were developed. ${ }^{23,24}$

Peters et al..$^{23}$ described one such system to deliver closed-loop stimulation in response to seizure detection. The system includes a Neoped 4000 EEG collection system (Nicolet BMSI, Madison, WI), two personal computers, custom software for electroencephalography (EEG) analysis using Visual $\mathrm{C}++$ (Version 6.0; Microsoft, Seattle, WA) and MATLAB (Version 5.0; Mathworks, Natick, MA), two Grass S12 stimulators (GrassTelefactor, West Warwick, RI), and other custom-built units to interface the components. A specific detection algorithm for this system using a wavelet transform and median filter was developed to detect events early and in real time. ${ }^{25}$ This algorithm results in earlier detection and shorter detection latencies compared to commercially available detection algorithms, which at the time were designed to detect the body of an event, and not the onset of an event. ${ }^{26}$ The median detection latency was $3.6 \mathrm{~s}$ for the generic algorithm. By adapting the filter to the indi- 
vidual subject's seizures, the detection latency was further reduced. In the five subjects with the greatest median detection delay, the adaptive approach reduced the median delay from 12.3 to $4.8 \mathrm{~s}$. In almost all cases in which the electrographic onset preceded the clinical onset, the detection occurred several tens of seconds before the clinical onset. ${ }^{27,28}$

The system was evaluated in a trial of eight patients undergoing intracranial monitoring. ${ }^{28}$ In four patients, responsive stimulation was delivered directly into the epileptogenic zone; in the other four patients, responsive stimulation was delivered into the anterior thalamic nucleus. With this study, the investigators demonstrated that automated responsive delivery of stimulation in close temporal proximity to the seizure onset was practicable. The study also provided preliminary evidence that high-frequency responsive stimulation could be efficacious, with three of four responders $(>50 \%$ seizure reduction) in patients who received stimulation to the epileptogenic zone and two of four responders in patients who received stimulation to the anterior thalamus.

Kossoff et al. ${ }^{24}$ also reported on experience using an external responsive neurostimulator. This open trial assessed clinical and electrographic effects of cortical stimulation applied to persons hospitalized and implanted with intracranial electrodes for purposes of localization. Stimulation was provided by an external device that detected electrographic seizures and applied preprogrammed stimulation. The detection tools (described in greater detail in the next section) were designed to require low computational power, so that they could be implemented in an implantable device. The detection tools are also highly configurable, to provide early and specific detections individualized for each patient.

The Kossoff et al. ${ }^{24}$ study reported on four patients who were treated with responsive stimulation for up to $68 \mathrm{~h}$ (range, 6-68 h) of responsive stimulation. In all patients, responsive stimulation appeared to be safe and well tolerated. Two patients reported brief transient side effects, such as sensations in the tongue, facial tingling, and visual flashes; however, the sensations stopped after stimulation parameters were changed. Although the study was not designed to assess efficacy, stimulation appeared to reduce the number of clinical seizures in both temporal and extratemporal locations, and electrographic seizures were altered and suppressed during the period when the patients received responsive stimulation. In one patient, stimulation also appeared to improve the baseline electrocorticography.

Evidence from these preliminary studies suggests that important parameters for responsive stimulation are temporal and spatial specificity. Early detection and accurate lead placement may thus be key to the success of responsive stimulation.

Whether chronic brain stimulation can exacerbate or initiate seizures in humans with epilepsy is not completely known. However, experience with DBS for Parkinson's disease and essential tremor and experience with vagus nerve stimulation for epilepsy have not raised clinical concern that these therapies kindle new seizure foci. Stimulation parameters for creating kindled seizures differ from the high-frequency, short-duration stimulation delivered to humans with movement disorders or epilepsy. In fact, this type of stimulation may provide what is called kindling antagonism, whereby stimulation in one area can inhibit the development of fully kindled seizures from another site. ${ }^{29}$

\section{THE NEUROPACE RNS SYSTEM}

Experience with the external responsive neurostimulator ${ }^{24}$ LED to the development of the first implantable responsive neurostimulator for epilepsy, the RNS system by neuropace. This system is capable of performing realtime seizure detection and delivering responsive electrical stimulation. At this time, the system is being evaluated in clinical trials to assess the safety and efficacy of responsive neurostimulation for the treatment of medically intractable partial-onset epilepsy.

The implantable components of the system include a cranially implanted neurostimulator, and intracranial depth and strip leads (FIG. 1). The neurostimulator is a battery-powered, microprocessor-controlled device that continuously monitors electrographic activity from the leads and delivers programmable electrical stimulation in response to detected events. Up to two leads, each containing four electrodes can be connected to the neurostimulator; the system can monitor and deliver responsive stimulation to two distinct epileptogenic zones independently.

The external components of the system include a physician programmer, a patient data transmitter, and a telemetry wand. The programmer is used by the physician to program detection and stimulation settings and retrieve stored information (e.g., electrographic activity) from the neurostimulator. The data transmitter is provided to the patient to allow uploading and remote monitoring of the device between clinic visits. The telemetry wand allows wireless communication between the neurostimulator and the programmer (or data transmitter). The device data and electrographic data are uploaded via the Internet to a central patient data management system and may be reviewed by physicians using a secure Web browser.

\section{Electrographic sensing and storage}

The neurostimulator continuously senses and monitors electrographic activity through the cortical depth and strip leads. Due to the constraints of an implanted system, the sensed electrographic data are not continuously recorded; however, the neurostimulator can store seg- 


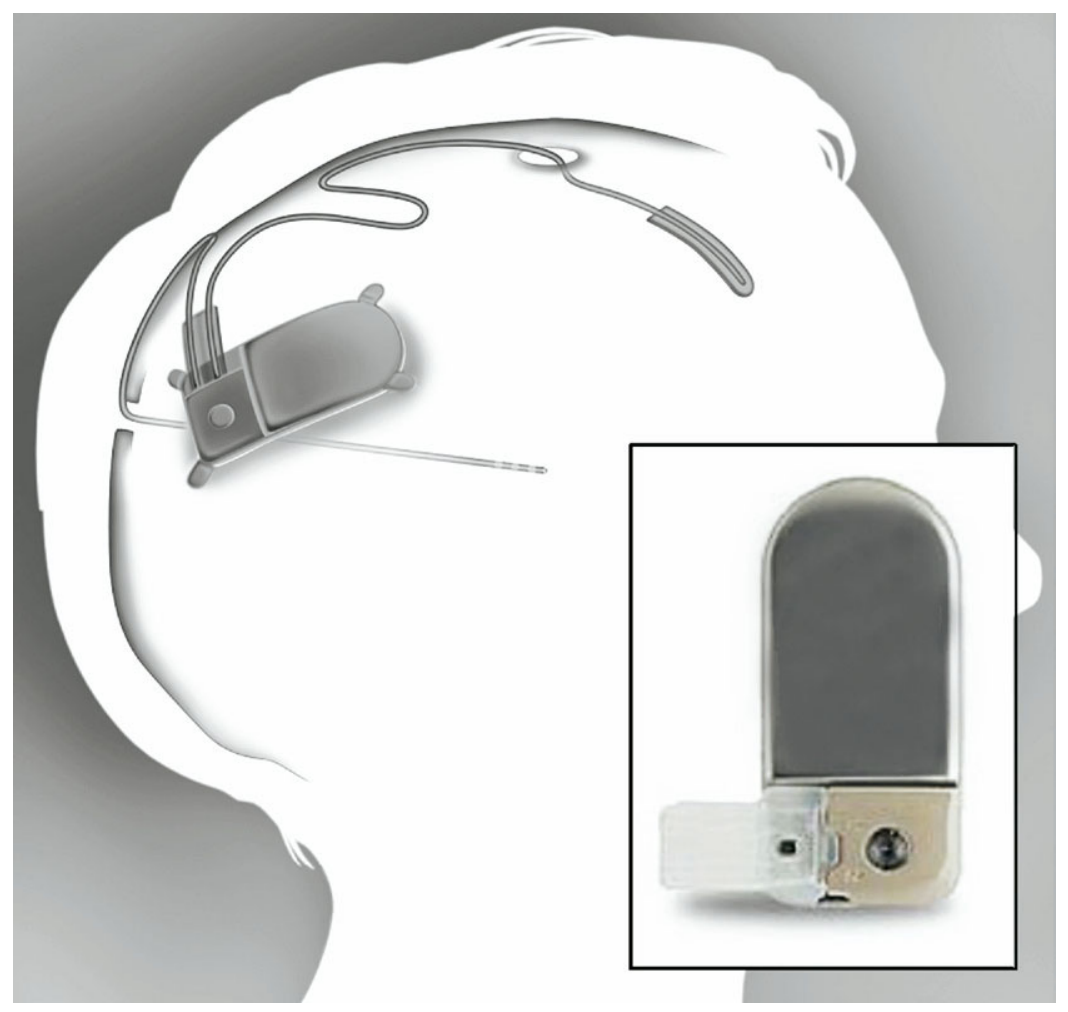

FIG. 1. Schematic illustration of the implanted RNS neurostimulator, depth lead, and cortical strip lead (NeuroPace, Mountain View, CA). The RNS neurostimulator (inset) is designed to match typical skull thickness and curvature, and is intended for implant in a ferrule, or socket, placed in a craniectomy. Up to two leads may be used with the system; each may be a depth lead or cortical (subdural) strip lead, and each has four electrode contacts, which are used for sensing and providing stimulation. To provide early seizure detection and delivery of focal electrical stimulation, leads are positioned using standard neurosurgical techniques as close as possible to the seizure focus or foci.

ments of the electrographic data for review by the physician. Electrographic data storage can be triggered by detection, responsive stimulation, scheduled time of day, magnet (used by the patient to indicate a clinical event), or other events as programmed by the physician. These data allow physicians to assess detection sensitivity and effects of stimulation.

\section{Detection}

The detection algorithms implemented in the RNS Neurostimulator system are designed to be computationally efficient and are highly optimized to perform real-time seizure detection within the constraints of currently available implantable technology, such as limited power and processing capabilities. Three detection tools are provided: area, line-length, and half-wave. The detection tools are highly configurable and can be adjusted by the physician to optimize the sensitivity and specificity trade-off for each individual patient. Up to two independent detectors can be programmed for any two sensing channels.

The half-wave tool, similar to that described by Gotman, ${ }^{30}$ is used to detect spikes and rhythmic activity occurring in specific frequency ranges. The half-wave tool segments the electrographic signal at local minima and maxima, resulting in half-waves, the amplitude and duration of which are representative of the amplitude and frequency components of the EEG. Half-waves that exceed a physician-programmed amplitude and duration are counted; the number of these half-waves occurring within a given window length must exceed a certain threshold for detection to occur. Detection parameters may be selected to adjust the sensitivity, specificity, and latency of the detection. For example, a detector may be configured for early detection (within a fraction of a second) with as few as two qualified half-waves, whereas a more specific detector may require several qualified half-waves that occur over a few seconds.

The line-length algorithm, described by Esteller et al., ${ }^{31,32}$ is used to identify changes in both amplitude and frequency. The line length is defined as the average of absolute sample-to-sample differences within a window. A short-term sliding window average (128 $\mathrm{ms}$ to $4 \mathrm{~s}$ ) is compared to a long-term sliding window average ( $4 \mathrm{~s}$ to $16 \mathrm{~min}$ ). A detection occurs when the short-term measurement exceeds an absolute or relative threshold, compared with the long-term measurement. A negative threshold can also be used to detect decreases in line length, which may represent a period of electrodecrement or decreased frequency. 


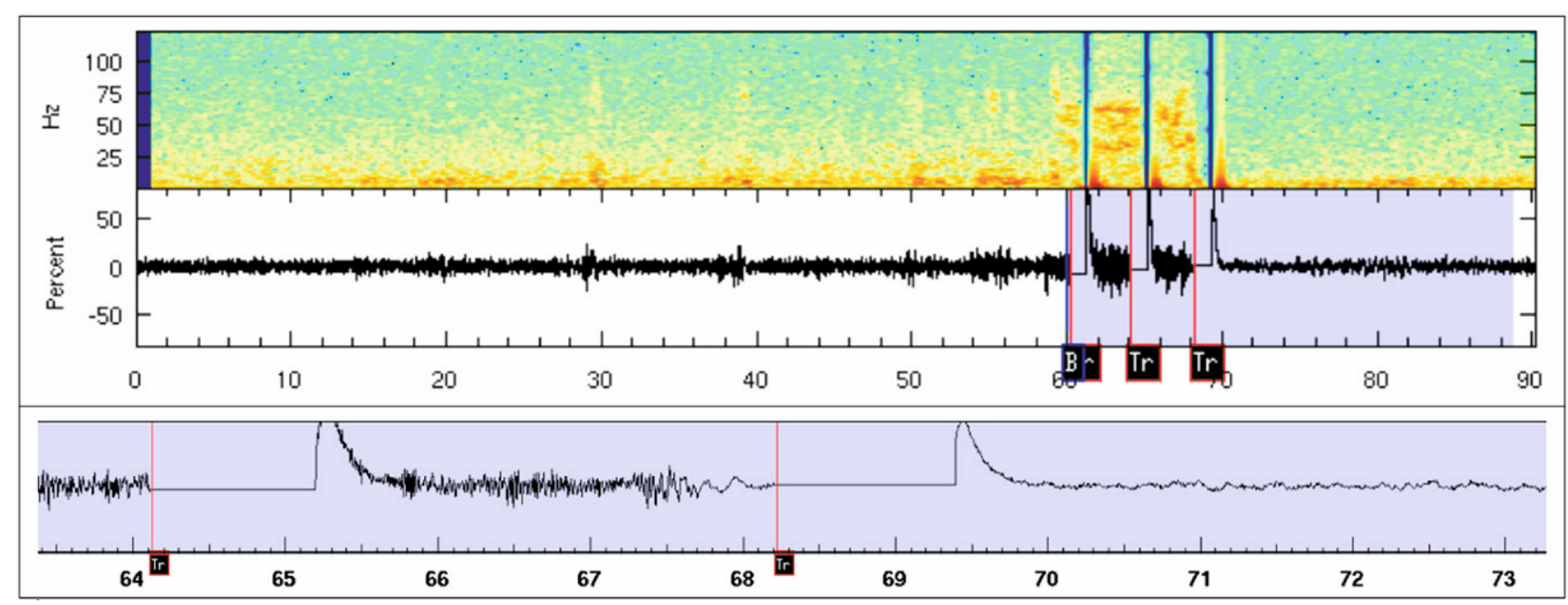

FIG. 2. Example of a possible seizure termination by responsive stimulation. The upper panel shows the fast Fourier transform spectrogram and time series for a single cortical electroencephalography channel. The lower panel shows a close-up of the time series around the time of termination. In the time series plots, detection is indicated by the vertical blue line and the ' $\mathrm{B}$ ' label, and responsive stimulation is indicated by the vertical red lines and the 'Tr' labels (the first is partly obscured by the ' $\mathrm{B}$ ' label).

The area feature, ${ }^{33}$ similar to an energy or power measurement, ${ }^{34,35}$ is used to identify changes in overall signal energy without regard for frequency. Area is defined as the average absolute area-under-the-curve within a window. As with line length, a short-term window average is compared to a long-term background window average, and detection occurs when a positive or negative threshold is exceeded. The area and line-length detection algorithms are efficient (requiring low computational power) and can be configured to detect events within a fraction of a second or to detect more subtle changes in amplitude, frequency, or power that occur over several seconds.

\section{Stimulation}

The neurostimulator delivers current-controlled, charge-balanced biphasic pulses; it can be programmed by the physician to deliver stimulation frequencies ranging from 1 to $333 \mathrm{~Hz}$, current amplitudes from 1 to 12 $\mathrm{mA}$, and pulse-widths from 40 to $1000 \mu \mathrm{s}$. The stimulation montage can be configured to deliver current between any combination of electrodes and the neurostimulator case.

Up to five individually configured sequential therapies of electrical stimulation may be programmed, where each therapy is composed of two independently configurable bursts (FIG. 2). The RNS will attempt to redetect the epileptiform activity after each therapy is delivered. If the epileptiform activity is still detected, the next (sequential) therapy will be delivered. If the epileptiform activity is no longer detected, the remaining therapies will not be delivered and the episode ends. The therapy sequence will refresh with the detection of each new episode.

Stimulation parameters are determined empirically, while respecting safety limits for charge density. According to studies of acute ${ }^{36,37}$ and chronic stimulation, ${ }^{38-41}$ the risks to the brain of electrical stimulation appear to be few so long as current densities are low enough to avoid tissue damage. The RNS system has controls in place at the programmer and neurostimulator so that current densities remain below a conservative limit of $25 \mu \mathrm{C} / \mathrm{cm}^{2}$ per phase. In addition, there is evidence that intermittent (more acute-like) stimulation poses fewer risks to neural tissue than does continuous stimulation. ${ }^{42-45}$

The stimulation configuration is also determined empirically. Options include providing bipolar stimulation across electrode pairs or stimulating across all eight electrodes to the case of the neurostimulator. Whether stimulation is delivered to a few or many electrodes depends to some extent on the area of onset.

\section{EXPERIENCE WITH THE RNS SYSTEM}

In a feasibility study of 65 patients, the NeuroPace RNS system demonstrated excellent safety and tolerability, as well as preliminary evidence of efficacy. Based on an interim analysis of 39 subjects, there were no serious device-related unanticipated adverse events. Stimulationrelated symptoms in several subjects were addressed by adjusting the stimulation settings. No subjects experienced sustained seizure types of greater severity or convulsive status epilepticus. There was preliminary evidence for efficacy in the initial 24 subjects with complete data. The responder rate ( $\geq 50 \%$ reduction in seizures) was $43 \%$ for complex partial seizures and 35\% for total disabling seizures (simple partial motor seizures, complex partial seizures, and secondarily generalized tonicclonic seizures). 
A randomized, double-blinded, multicenter, shamcontrolled clinical trial is currently underway to establish whether the RNS System is safe and effective as an adjunctive therapy for medically refractory partial-onset epilepsy (Clinicaltrials.gov NCT00264810). The investigation will enroll 240 adult subjects (18-70 years of age) with medically refractory partial epilepsy who have an average of three or more disabling simple partial motor seizures, complex partial seizures, or secondarily generalized seizures per 28 days.

\section{CONCLUSIONS}

There is growing evidence that responsive neurostimulation may be a safe and effective treatment option for epilepsy as animal and human data support the concept that responsive stimulation can abort epileptiform activity. Moreover, responsive stimulation may offer advantages to current therapy options due to the specificity of the therapy. In contrast to pharmacotherapy, which may cause concomitant side effects due to the effect on the system, electrical stimulation may be targeted to the specific brain regions involved in the seizure. In addition, responsive stimulation provides specificity in terms of time. Treatment is provided as needed, potentially reducing the likelihood of functional disruption or habituation due to continuous treatment. The RNS system offers the first opportunity to directly assess the efficacy of chronic responsive stimulation in a controlled trial. Trials thus far have demonstrated the safety of the technique, but completion of well-designed clinical trials currently in progress is necessary to assess efficacy.

\section{REFERENCES}

1. Begley CE, Famulari M, Annegers JF, et al. The cost of epilepsy in the United States: An estimate from population-based clinical and survey data. Epilepsia 2000;41:342-351.

2. Brodie MJ, Dichter MA. Antiepileptic drugs. N Engl J Med 1996; 334:168-175.

3. Morrell MJ. Antiepileptic medications for the treatment of epilepsy. Semin Neurol 2002;22:247-258.

4. Engel J Jr, Wiebe S, French J, et al. Practice parameter: temporal lobe and localized neocortical resections for epilepsy: report of the Quality Standards Subcommittee of the American Academy of Neurology, in association with the American Epilepsy Society and the American Association of Neurological Surgeons [Erratum in: Neurology 2003;60:1396]. Neurology 2003;60:538-547.

5. Fisher RS, Handforth A. Reassessment: vagus nerve stimulation for epilepsy: a report of the Therapeutics and Technology Assessment Subcommittee of the American Academy of Neurology. Neurology 1999;53:666-669.

6. Penfield W, Jasper H. Electrocorticography. In: Epilepsy and the functional anatomy of the human brain. Boston: Little, Brown, 1954:692-738.

7. Oommen J, Morrell M, Fisher RS. Experimental electrical stimulation therapy for epilepsy. Curr Treat Options Neurol 2005;7:261271.

8. Morrell M. Brain stimulation for epilepsy: can scheduled or responsive neurostimulation stop seizures? Curr Opin Neurol 2006; 19:164-168.

9. Li Y, Mogul DJ. Electrical control of epileptic seizures. J Clin Neurophysiol 2007;24:197-204.
10. Cooper IS, Amin I, Riklan M, Waltz JM, Poon TP. Chronic cerebellar stimulation in epilepsy: clinical and anatomical studies. Arch Neurol 1976;33:559-570.

11. Cooper IS, Upton AR. Therapeutic implications of modulation of metabolism and functional activity of cerebral cortex by chronic stimulation of cerebellum and thalamus. Biol Psychiatry 1985;20: $811-813$.

12. Chkhenkeli SA, Chkhenkeli IS. Effects of therapeutic stimulation of nucleus caudatus on epileptic electrical activity of brain in patients with intractable epilepsy. Stereotact Funct Neurosurg 1997;69:221-224.

13. Elisevich K, Jenrow K, Schuh L, Smith B. Long-term electrical stimulation-induced inhibition of partial epilepsy: case report. J Neurosurg 2006;105:894-897.

14. Velasco M, Velasco F, Velasco AL, et al. Subacute electrical stimulation of the hippocampus blocks intractable temporal lobe seizures and paroxysmal EEG activities. Epilepsia 2000;41:158169.

15. Vonck K, Boon P, Goossens L, et al. Neurostimulation for refractory epilepsy. Acta Neurol Belg 2003;103:213-217.

16. Halpern CH, Samadani U, Litt B, Jaggi JL, Baltuch GH. Deep brain stimulation for epilepsy. Neurotherapeutics 2008;5:000-000 (this issue).

17. Psatta DM. Control of chronic experimental focal epilepsy by feedback caudatum stimulations. Epilepsia 1983;24:444-454.

18. Nakagawa M, Durand D. Suppression of spontaneous epileptiform activity with applied currents. Brain Res 1991;567:241-247.

19. Kayyali H, Durand D. Effects of applied currents on epileptiform bursts in vitro. Exp Neurol 1991;113:249-254.

20. Warren RJ, Durand DM. Effects of applied currents on spontaneous epileptiform activity induced by low calcium in the rat hippocampus. Brain Res 1998;806:186-195.

21. Lesser RP, Kim SH, Beyderman L, et al. Brief bursts of pulse stimulation terminate afterdischarges caused by cortical stimulation. Neurology 1999;53:2073-2081.

22. Motamedi GK, Lesser RP, Miglioretti DL, et al. Optimizing parameters for terminating cortical afterdischarges with pulse stimulation [Erratum in: Epilepsia 2002;43:1441]. Epilepsia 2002;43: $836-846$

23. Peters TE, Bhavaraju NC, Frei MG, Osorio I. Network system for automated seizure detection and contingent delivery of therapy. J Clin Neurophysiol 2001;18:545-549.

24. Kossoff EH, Ritzl EK, Politsky JM, et al. Effect of an external responsive neurostimulator on seizures and electrographic discharges during subdural electrode monitoring. Epilepsia 2004;45: $1560-1567$.

25. Osorio I, Frei MG, Wilkinson SB. Real-time automated detection and quantitative analysis of seizures and short-term prediction of clinical onset. Epilepsia 1998;39:615-627.

26. Gotman J. Automatic seizure detection: improvements and evaluation. Electroencephalogr Clin Neurophysiol 1990;76:317-324.

27. Osorio I, Frei MG, Giftakis J, et al. Performance reassessment of a real-time seizure-detection algorithm on long ECoG series. Epilepsia 2002;43:1522-1535.

28. Osorio I, Frei MG, Sunderam S, et al. Automated seizure abatement in humans using electrical stimulation. Ann Neurol 2005;57: $258-268$

29. Bertram E. The relevance of kindling for human epilepsy. Epilepsia 2007;48 Suppl 2:65-74.

30. Gotman J. Automatic recognition of epileptic seizures in the EEG. Electroencephalogr Clin Neurophysiol 1982;54:530-540.

31. D'Alessandro M, Esteller R, Vachtsevanos G, Hinson A, Echauz J, Litt B. Epileptic seizure prediction using hybrid feature selection over multiple intracranial EEG electrode contacts: a report of four patients [Erratum in: IEEE Trans Biomed Eng 2003;50:1041]. IEEE Trans Biomed Eng 2003;50:603-615.

32. Esteller R, Echauz J, Tcheng T, Litt B, Pless B. Line length: an efficient feature for seizure onset detection. Engineering in Medicine and Biology, 2001, EMBS ‘01. Proc Annu Int Conf IEEE 23rd 2001;2:1707-1710.

33. Echauz J, Padovani DA, Esteller R, Litt B, Vachtsevanos G. Median-based filtering methods for EEG seizure detection. Proc Joint BMES/IEEE-EMBS Conf 1st, Atlanta, GA, 1999;1:439. 
34. Litt B, Esteller R, Echauz J, et al. Epileptic seizures may begin hours in advance of clinical onset: a report of five patients. Neuron 2001;30:51-64.

35. Litt B, Esteller R, D’Alessandro M, et al. Evolution of accumulated energy predicts seizures in mesial temporal lobe epilepsy. Proc Joint BMES/IEEE-EMBS Conf 1st, Atlanta, GA, 1999;1:440.

36. Lesser RP, Lüders H, Klem G, et al. Extraoperative cortical functional localization in patients with epilepsy. J Clin Neurophysiol 1987;4:27-53.

37. Risinger MW, Gumnit RJ. Intracranial electrophysiologic studies. Neuroimaging Clin N Am 1995;5:559-573.

38. Haberler C, Alesch F, Mazal PR, et al. No tissue damage by chronic deep brain stimulation in Parkinson's disease. Ann Neurol 2000;48:372-376.

39. Nguyen JP, Lefaucheur JP, Decq P, et al. Chronic motor cortex stimulation in the treatment of central and neuropathic pain: correlations between clinical, electrophysiological and anatomical data. Pain 1999;82:245-251.
40. Ebel H, Rust D, Tronnier V, Boker D, Kunze S. Chronic precentral stimulation in trigeminal neuropathic pain. Acta Neurochir (Wien) 1996;138:1300-1306.

41. Tsubokawa T, Katayama Y, Yamamoto T, Hirayama T, Koyama S. Chronic motor cortex stimulation in patients with thalamic pain. J Neurosurg 1993;78:393-401.

42. Bartlett JR, Doty RW Sr, Lee BB, Negrão N, Overman WH Jr. Deleterious effects of prolonged electrical excitation of striate cortex in macaques. Brain Behav Evol 1977;14:46-66.

43. Dauth G, Defendini R, Gilman S, Tennyson V, Kremzner L. Longterm surface stimulation of the cerebellum in the monkey: I. Light microscopic, electrophysiologic, and clinical observations. Surg Neurol 1977;7:377-384.

44. Gordon B, Lesser RP, Rance NE, et al. Parameters for direct cortical electrical stimulation in the human: histopathologic confirmation. Electroencephalogr Clin Neurophysiol 1990;75:371-377.

45. Lesser R, Gordon B, Uematsu S. Electrical stimulation and language. J Clin Neurophysiol 1994;11:191-204. 